\title{
AVALIAÇÃO SENSORIAL DE AMOSTRAS COMERCIAIS DE LEITE CONDENSADO
}

\section{Sensory evaluation of commercial samples of condensed milk}

\author{
Ana Laís Andrade Gaspardi , Jéssika Michelli ${ }^{1}$, Clara Gonçalves de Pontes ${ }^{1}$, \\ Lara de Andrade Oliveira ${ }^{1}$, Natali Alcântara Brandão ${ }^{1}$, Mariana Borges de Lima Dutra ${ }^{1 *}$
}

\section{RESUMO}

O Brasil se destaca pela grande produção e consumo de leite condensado. As indústrias nacionais possuem alta tecnologia de fabricação, oferecendo produtos com alto valor agregado e possibilitando ao consumidor diversas opções de marcas, preços e qualidade. Este trabalho teve como objetivo verificar se existe diferença sensorial (ao nível de 5\% de significância) quanto à aceitação de 6 amostras de leite condensado comerciais. As amostras foram avaliadas por 120 consumidores, recrutados voluntariamente, pelo teste de aceitação. Os resultados da ANOVA mostraram que as amostras C, D, E e F apresentaram aceitação significativamente superior $(\mathrm{p} \leq 0,05)$ às demais na escala hedônica, enquanto a amostra A obteve menor aceitação em relação a todos os atributos relacionados na escala hedônica. $\mathrm{O}$ teste de intenção de compra demonstrou que as amostras $\mathrm{C}$ e $\mathrm{F}$ obtiveram as maiores frequências de intenção de compra positiva. O Mapa de Preferência Interno confirmou os resultados da ANOVA e do teste de intenção de compra indicando uma maior preferência dos provadores pelas amostras $\mathrm{C}$ e F. As amostras adquiridas foram fabricadas com os mesmos ingredientes, no entanto, pressupõe-se que as diferenças sensoriais ocorreram devido a qualidade das matérias-primas utilizadas e devido aos diversos processos de elaboração do produto de cada empresa. Este fato prova que é necessário controlar a qualidade da matéria-prima e o tipo de processo utilizado para a obtenção do produto, pois estes parâmetros podem influenciar a aceitação sensorial dos consumidores. A qualidade e a padronização do produto final é um fator primordial para manter a produtividade e a competitividade de mercado.

Palavras-chave: teste de aceitação, leite condensado, mapa de preferência interno.

1 Instituto Federal de Educação, Ciência e Tecnologia do Sul de Minas Gerais, Campus Inconfidentes, Praça Tiradentes, 416, Centro, 37576-000, Inconfidentes, MG, Brasil. E-mail: mariana.dutra@ ifsuldeminas.edu.br

* Autor para correspondência.

Recebido / Received: 31/08/2015

Aprovado / Approved: 19/09/2016 


\begin{abstract}
The Brazil stands out for the huge production and consumption of condensed milk, industries rely on high-tech production, offering products with high added value and enabling the consumer to purchase products from different brands, prices and quality. This job aimed to verify if there is sensory difference at the $5 \%$ level of significance as the acceptance of 6 samples of commercial condensed milk. The samples were evaluated by 120 consumers, recruited voluntarily for the acceptance test. The results of ANOVA showed that the samples C, D, E and F were significantly higher acceptance $(\mathrm{p}=0.05)$ in the other hedonic scale. While the sample A had lower acceptance for all the attributes listed on the hedonic scale. The purchase intention test showed that the $\mathrm{C}$ and $\mathrm{F}$ samples achieved the highest frequencies of positive purchase intention. The internal preference mapping confirmed the results of the ANOVA and purchase intention test indicating a greater preference of tasters by $\mathrm{C}$ and $\mathrm{F}$ samples. Acquired samples were made with the same ingredients, it is assumed that the sensory differences occurred due to the quality of raw material and to product development processes in each company. It is necessary to identify the importance of quality of the raw material and the type of process used to obtain the product enabling the improvement of brands less accepted in its production, increasing the acceptance and quality of their own product. The quality along with the standardization of the final product is a key factor for maintaining competitiveness and increasing productivity in the market.
\end{abstract}

Keywords: acceptance testing, condensed milk, internal preference map.

\section{INTRODUÇ̃̃O}

O leite condensado surgiu com as experiências do francês Nicolas Appert (1820), na esterilização e conservação de alimentos em embalagens herméticas. Mais tarde, o empreendedor norte-americano Gail Borden, tentando desidratar o leite de vaca comum, descobriu que, antes de transformar-se em leite em pó, o produto se transformava em leite condensado. A descoberta de Gail Borden foi patenteada no ano de 1856 e só foi valorizada quatro anos depois. A partir daí, o consumo de leite condensado obteve um aumento (GALINA, 2010).

O RIISPOA (BRASIL, 1952) define leite condensado ou "leite condensado com açúcar" como um produto resultante da desidratação em condições próprias do leite adicionado de açúcar.
O Brasil é o país que mais consome leite condensado no mundo, são 200 mil toneladas por ano. O leite condensado tem boa receptividade em vários países, principalmente os árabes. O produto brasileiro destaca-se pela qualidade, pela escala de produção de alto padrão tecnológico e por oferecer valor agregado (GALINA, 2010).

Nas últimas décadas se intensificou o estudo dos produtos através de um painel sensorial, pois a qualidade sensorial pode ser considerada um fator chave na aceitação de um produto alimentício. Os alimentos evocam sensações diversas de sabor, odor, aroma, textura, dor, frio, calor, as quais podem ser mensuradas por métodos sensoriais (CARDELLO, 1996; RETONDO, 2004).

A análise sensorial é definida pela Associação Brasileira de Normas Técnicas (ABNT, 1993) como a disciplina científica 
usada para evocar, medir, analisar e interpretar reações das características dos alimentos e materiais como são percebidas pelos sentidos da visão, olfato, gosto, tato e audição.

Os testes sensoriais utilizam os órgãos dos sentidos humanos como "instrumentos" de medida e possui importante vantagem como, por exemplo, determinar a aceitação de um produto por parte dos consumidores (CARDELLO; CARDELLO, 1998). Segundo Marcellini (2005) a análise de aceitação é de extrema importância por refletir o grau que os consumidores gostam ou desgostam de um produto.

O leite condensado é um produto bastante popular no Brasil, servido como acompanhamento de frutas e sorvetes, também muito utilizado no preparo de sobremesas em geral, tortas, mousses, caldas, vitaminas, coquetéis entre outros (FLAUZINO, 2007). Além disso, o conhecimento de propriedades sensoriais de leite condensado e a aceitação pelos consumidores, levando-se em consideração o tipo de produto preferido, ainda é desconhecido.

Assim, objetivou-se com o presente estudo avaliar sensorialmente seis marcas de leite condensado encontrados no mercado varejista das cidades de Inconfidentes (MG) e Pouso Alegre (MG).

\section{MATERIAL E MÉTODOS}

Foram avaliadas seis marcas comerciais de leite condensado. Os produtos foram adquiridos em supermercados dos municípios de Inconfidentes e Pouso Alegre, no estado de Minas Gerais. Todas as amostras foram elaboradas com leite integral, açúcar e lactose conforme informações contidas nos rótulos.

O teste de aceitação e intenção de compra foi realizado no Instituto Federal de Educação, Ciência e Tecnologia do Sul de Minas Gerais - Campus Inconfidentes, e contou com a participação de 120 consumidores sendo 66 homens e 54 mulheres, com idade de 14 a 60 anos. A realização do teste de aceitação foi divulgada por comunicação verbal e todos interessados participaram voluntariamente.

As amostras de leite condensado foram apresentadas aos consumidores a temperatura ambiente, em copos plásticos descartáveis codificados com algarismos aleatórios de 3 dígitos e em quantidade de cerca de $10 \mathrm{~mL}$, de forma monádica utilizando delineamento em bloco completo balanceado. Foi orientado aos provadores que tomassem água entre as amostras oferecidas, evitando a fadiga sensorial.

As formulações foram avaliadas por meio do teste de aceitação sensorial para os atributos: aparência, aroma, sabor, textura e impressão global. Para o teste, foi adotada a escala hedônica estruturada de nove pontos ancorada nos extremos por "desgostei muitíssimo" e "gostei muitíssimo" (STONE; SIDEL, 2004). Os consumidores também avaliaram a intenção de compra do produto, utilizando-se escala de cinco pontos variando de "certamente não compraria" a "certamente compraria” (MEILGAARD et al., 1999).

Também foi aplicada a análise por mapa de preferência interno com os dados de impressão global do teste de aceitação.

A análise dos resultados do teste de aceitação foi realizada por meio do software Sensomaker ${ }^{\circledR}$ (PINHEIRO et al., 2013).

\section{RESULTADOS E DISCUSSÃO}

As médias das notas dadas pelos consumidores para os atributos aparência, aroma, sabor, textura e impressão global avaliados no teste de aceitação estão apresentados na Tabela 1.

Não houve diferença significativa $(\mathrm{p}>0,05)$ para as amostras C, D, E e F de leite condensado para os atributos aparência, textura e impressão global, sendo essas amostras as que apresentaram maior aceitação para esses atributos. A amostra B não diferiu $(\mathrm{p} \leq 0,05)$ das amostras C, D, E e F para o 
atributo impressão global. Para o atributo aroma, as amostras mais aceitas $(\mathrm{p} \leq 0,05)$ foram $\mathrm{C}$ e $\mathrm{F}$, já para o sabor as amostras mais aceitas $(p \leq 0,05)$ foram D e F. A amostra A foi a menos aceita $(p \leq 0,05)$ em todos os atributos avaliados. A Figura 1 apresenta a frequência das respostas de intenção de compra do leite condensado.

Os resultados de intenção de compra demonstraram que as amostras de leite condensado $\mathrm{F}$ e C obtiveram as maiores frequências de intenção de compra positiva, correspondendo a resposta "certamente compraria" totalizando $47 \%$ e $38 \%$ respectivamente, o que pode estar relacionado com a maior aceitação dessas amostras. A amostra D também apresentou resultados significativos para a intenção de compra positiva, correspondendo a maior frequência para a resposta provavelmente compraria (38\%).

A amostra B apresentou maior frequência $(30 \%)$ de indecisão na intenção de compra representada pelo termo "talvez compraria". As amostras A, C e D apresentaram a mesma frequência de indecisão de compra (28\%). A amostra A apresentou maior frequência de intenção de compra negativa que corresponde as atitudes de compra "provavelmente não compraria" e "certamente não compraria", correspondendo a $30 \%$ e $19 \%$ respectivamente.

O mapa de preferência interno está apresentado na Figura 2, onde os pontos repre-

Tabela 1 - Valores médios* dos atributos do teste de aceitação das amostras de leite condensado

\begin{tabular}{cccccc}
\hline Amostras & Aparência & Aroma & Sabor & Textura & $\begin{array}{c}\text { Impressão } \\
\text { Global }\end{array}$ \\
\hline Amostra A & $5,79^{\mathrm{c}}$ & $6,91^{\mathrm{c}}$ & $6,45^{\mathrm{c}}$ & $5,56^{\mathrm{c}}$ & $5,48^{\mathrm{b}}$ \\
Amostra B & $6,69^{\mathrm{b}}$ & $7,43^{\mathrm{b}}$ & $6,86 \mathrm{~b}^{\mathrm{c}}$ & $6,53^{\mathrm{b}}$ & $7,07^{\mathrm{a}}$ \\
Amostra C & $7,38^{\mathrm{a}}$ & $8,01^{\mathrm{a}}$ & $7,32^{\mathrm{ab}}$ & $7,46^{\mathrm{a}}$ & $7,42^{\mathrm{a}}$ \\
Amostra D & $7,48^{\mathrm{a}}$ & $7,89^{\mathrm{ab}}$ & $7,48^{\mathrm{a}}$ & $7,46^{\mathrm{a}}$ & $7,2^{\mathrm{a}}$ \\
Amostra E & $7,27^{\mathrm{a}}$ & $7,713^{\mathrm{ab}}$ & $7,31^{\mathrm{ab}}$ & $7,35^{\mathrm{a}}$ & $7,18^{\mathrm{a}}$ \\
Amostra F & $7,78^{\mathrm{a}}$ & $7,98^{\mathrm{a}}$ & $7,57^{\mathrm{a}}$ & $7,85^{\mathrm{a}}$ & $7,63^{\mathrm{a}}$ \\
\hline
\end{tabular}

*Médias seguidas pela mesma letra, numa mesma coluna, não diferem entre si a $\mathrm{p} \leq 0,05$ pelo teste Tukey.

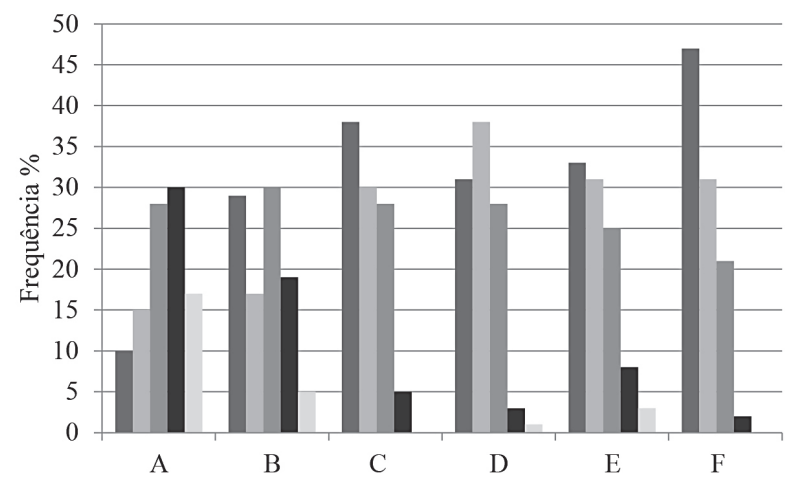

घCertamente compraria

$\square$ Provavelmente compraria

$\square$ Talvez compraria

• Provavelmente não compraria

$\square$ Certamente não compraria

Figura 1 - Distribuição da frequência de respostas de intenção de compra das amostras de leite condensado 
sentam as amostras e os vetores representam os consumidores. No mapa cada vetor representa um provador avaliando a resposta para o atributo impressão global, considerando as referências individuais de cada um dos 120 consumidores.

As amostras $\mathrm{C}$ e $\mathrm{F}$ (destacadas em vermelho na Figura 2) apresentam maior concentração vetores (consumidores) direcionados a elas. Esta preferência pode ser confirmada pelo teste Tukey (Tabela 1), onde estas amostras foram as que apresentaram maiores médias.

As amostras D e E apresentaram aceitação intermediária o que pode ser observado devido a menor concentração de vetores (consumidores) direcionados a elas se comparada às amostras $\mathrm{C}$ e $\mathrm{F}$ com maior aceitação. Por fim, percebe-se uma grande distância entre as amostras A e B e as mais aceitas e também uma pequena quantidade de vetores direcionados a elas, podendo considerar as amostras A e B as menos aceitas pelos consumidores.

As amostras de leite condensado foram elaboradas com os mesmos ingredientes, desta forma supõe-se que a divergência encontrada no teste de aceitação do leite condensado pode-se dar pela qualidade da matéria prima utilizada ou pelo tipo de processamento aplicado pelas indústrias na elaboração dos produtos.

As amostras mais aceitas não possuem relação com as marcas de maior valor ou mais populares. Neste estudo não foi demonstrado o valor de compra de cada amostra, no entanto, foi observado que as marcas mais caras não estiveram entre as mais aceitas, porém também não foram as que apresentaram maior rejeição.

\section{CONCLUSÕES}

Os resultados obtidos indicaram que os consumidores apresentaram uma maior aceitação pelas amostras $\mathrm{C}$ e $\mathrm{F}$, o que pode ser observado pelas maiores médias no teste de Tukey ( $\mathrm{p} \leq 0,05)$ e pelas maiores frequências de intenção de compra positiva, podendo ser confirmados os resultados pelo mapa de preferência interno. Em destaque, a amostra A foi a menos aceita, pois apresentou alto

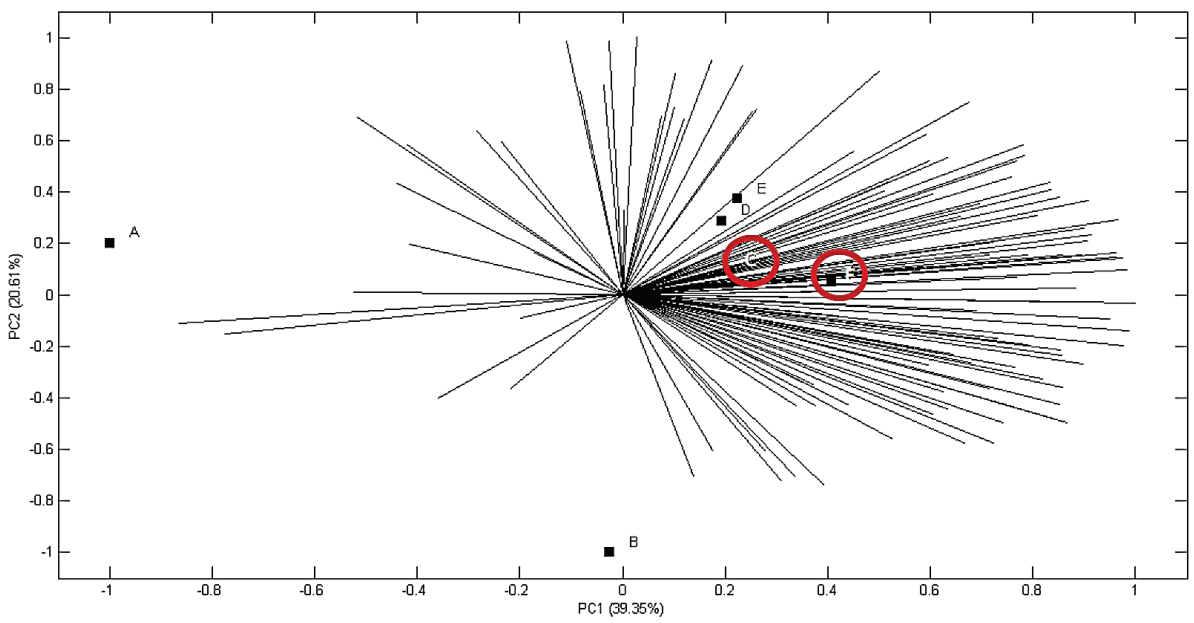

Figura 2 - Mapa de preferência interno para as amostras de leite condensado. Amostras $\mathrm{C}$ e $\mathrm{F}$ (destacadas em vermelho) foram as mais preferidas 
índice de rejeição, nos testes de aceitação e de intenção compra. O mapa de preferência interno confirmou os resultados obtidos pela ANOVA e ainda possibilitou observar claramente a segmentação dos consumidores, em função das características sensoriais das amostras. Uma vez que todas as amostras de leite condensado foram elaboradas com os mesmos ingredientes, supõem-se que as diferenças sensoriais podem se dar devido a qualidade da matéria prima e ao processo de fabricação que cada empresa utiliza.

Assim, seria necessário identificar cada matéria prima e processo utilizado para a fabricação de cada de leite condensado para então obter-se uma relação com a aceitação dessas amostras por parte dos consumidores, possibilitando que as empresas das marcas menos aceitas possam melhorar sua produção e assim aumentar a aceitação de seu produto.

\section{REFERÊNCIAS}

ASSOCIAÇÃO BRASILEIRA DE NORMAS TÉCNICAS. NBR 12806: análise sensorial dos alimentos e bebidas: terminologia. 1993. 8 p.

BRASIL. Ministério da Agricultura. R.I.I.S.P.O.A. Regulamento da Inspeção Industrial e Sanitária de Produtos de Origem Animal (Aprovado pelo Decreto $n^{\circ}$ 30.691 , de 20.03.52, alterado pelo decreto $\mathrm{n}^{\mathrm{o}} 1255$, de 25.06.52). Diário Oficial da República Federativa do Brasil, Brasília, 29 mar. 1952.

CARDELlO, H. M. A. B. Caracterização sensorial de aspartame, ciclamato/sacarina 2:1 extrato de folhas de estévia (Stévia rebaudiana Bertoni): equivalências em doçura, análise descritiva quantitativa e análise tempo-intensidade. 1996. 237 p. Tese (Doutorado em Engenharia de Alimentos) - Universidade Estadual de Campinas, Campinas, 1996.
CARDELLO, H. M. A.; CARDELLO, L. Teor de vitamina $C$, atividade de ascorbato oxidase e perfil sensorial de manga (Mangífera índica L.) var. haden, durante o amadurecimento. Ciência e Tecnologia de Alimentos, v. 18, n. 2, p. 211-217, 1998.

FLAUZINO, R. D. Influência da temperatura e do teor de gordura nos parâmetros reológicos do leite condensado e creme de leite. 2007. 100 p. Dissertação (Mestrado em Engenharia e Ciência de Alimentos) - Universidade Estadual Paulista Júlio de Mesquita Filho, São José do Rio Preto, 2007.

GALINA, C. Produção de Leite Condensado. Blumenau, 2010. 166 p.

MARCELLINI, P. S. Caracterização sensorial por perfil livre e análise tempo intensidade de suco de abacaxi (Ananas comosus (L.) Merril) reconstituído e adoçado com diferentes edulcorantes. 2005. 85 p. Tese (Doutorado em Engenharia de Alimentos) - Universidade Estadual de Campinas, Campinas, 2005.

MEILGAARD, M.; CIVILLE, G. V.; CARR, B. T. Sensory Evaluation Techniques. New York: Boca Raton, $3^{\mathrm{a}}$ ed. 1999. 387p.

PINHEIRO, A. C. M.; NUNES, C. A.; VIETORIS, V. SensoMaker: a tool for sensorial characterization of food products. Ciência e Agrotecnologia, v. 37, n. 3, p. 199-201, 2013.

RETONDO, C. G. Química das sensações: desenvolvimento de um material didático interdisciplinar para o ensino superior. 2004. 282 p. Dissertação (Mestrado em Química) - Universidade Estadual de Campinas, Campinas, 2004.

STONE, H.; SIDEL, J. Sensory evaluation practices. $3^{\mathrm{a}}$ ed. New York: Academic Press, 2004. 408 p. 